\title{
La création des IUFM et la recherche en éducation : témoignages et réflexions
}

\section{Michèle Verdelhan-Bourgade et Pierre Boutan}

\section{(2) OpenEdition}

12 Journals

Édition électronique

URL : http://journals.openedition.org/trema/2926

DOI : 10.4000/trema.2926

ISSN : 2107-0997

Éditeur

Faculté d'Éducation de l'université de Montpellier

\section{Édition imprimée}

Date de publication : 1 juin 2013

Pagination : 9 - 21

ISBN : 1167-315X

ISSN : 1167-315X

\section{Référence électronique}

Michèle Verdelhan-Bourgade et Pierre Boutan, « La création des IUFM et la recherche en éducation témoignages et réflexions », Tréma [En ligne], 39|2013, mis en ligne le 01 juin 2015, consulté le 19 avril 2019. URL : http://journals.openedition.org/trema/2926 ; DOI : 10.4000/trema.2926

Ce document a été généré automatiquement le 19 avril 2019

Trema 


\title{
La création des IUFM et la recherche en éducation : témoignages et réflexions
}

\author{
Michèle Verdelhan-Bourgade et Pierre Boutan
}

\section{NOTE DE L'ÉDITEUR}

* Témoins et acteurs de toute la période qui précède la création des IUFM, puis de leurs dix premières années, les auteurs ont une expérience à la fois commune et diversifiée, tant comme représentants des personnels que participants à des équipes relevant de la recherche en éducation.

Ils travaillent ensemble actuellement sur les manuels scolaires, notamment ceux des pays méditerranéens (site http://rimamed.fr).

\section{IUFM et recherche : un processus qui vient de loin}

1 Pourquoi et comment améliorer l'instruction et l'éducation de tous les enfants? Contrairement aux apparences, se poser ces questions ne va nullement de soi, si on se place dans une perspective historique à long terme. On peut d'autant mieux l'admettre que l'on touche ici aux finalités de l'éducation, ce qui concerne l'ensemble de la société. À plus courte distance dans le temps, ces mêmes questions ont suscité et suscitent toujours de vifs débats, que la création des Instituts Universitaires de Formation des Maîtres, annoncée dans la loi de programmation de 1989, n'a pas manqué d'amplifier. En effet, cette nouvelle institution s'inscrivait dans une longue séquence historique, où, un peu plus d'un siècle après l'enseignement secondaire classique ${ }^{1}$, c'était au tour de l'ensemble de l'enseignement secondaire (technique et professionnel), de l'ensemble de l'enseignement primaire de se voir aussi dépendre de l'enseignement supérieur, et qui plus est dans le même établissement. Qui disait enseignement supérieur, disait contact 
avec le savoir en train de se faire, donc avec la recherche. On laissera ici de côté la question des problèmes institutionnels qui se posèrent, pour s'en tenir à un seul, celui du rapport à la recherche.

2 En parvenant à cette création, qui arrivait au terme de dizaines d'années de luttes, les forces progressistes qui étaient à son initiative pensaient avoir fait le plus difficile. Elles pouvaient en outre considérer, de par leur expérience, que l'on pourrait s'appuyer sur la recherche en matière d'éducation, portée à la fois par les travaux internationaux (en particulier américains) et une certaine tradition française, depuis l'œuvre d'Alfred Binet dès avant 1914, jusqu'à ceux conduits par l'Institut Pédagogique National, devenu Institut National de la Recherche Pédagogique, de la fin des années 1960 au début des années 1980. Certes, mettre en œuvre une démarche de recherche dans ce domaine, où plusieurs champs de connaissances étaient concernés, ne pouvait pas ne pas poser de problèmes. L'environnement francophone (Belgique, Suisse, Canada) incitait également à légitimer une démarche de cette nature, sans doute non exempte du risque de positivisme, mais qui évitait de voir s'affronter des affirmations de principe sans effort de rationalisation.

Ce qui faisait une autre originalité de ces travaux de l'IPN puis de l'INRP, c'était qu'un certain nombre concernait des disciplines scolaires communes au primaire et au secondaire. On parlait alors de "recherche-action", sous la direction de Louis Legrand notamment. En fonction d'une commande ministérielle visant à remettre en cause les cursus, dès lors que la scolarité obligatoire était portée à 16 ans à partir de la génération d'élèves sortant du primaire en 1959, une commission dite Rouchette (du nom de l'inspecteur général qui la présidait) allait à la fin des années 1960 produire pour l'enseignement du français un "plan de rénovation", dont l'originalité venait de la volonté d'expérimenter de nouvelles instructions avant de les communiquer au public. En prolongeant la commission Rouchette dans l'ensemble de la nouvelle école obligatoire (jusqu'à 16 ans), donc en englobant le secondaire, par la commission Chevalier, la primauté de la dimension des contenus disciplinaires était affirmée. D’autres commissions, concernant aussi le secondaire, vont être mises en place avec le même but dans les autres disciplines (mathématiques, avec la création des Instituts de recherche pour l'enseignement des mathématiques - IREM - en 1969, sciences - commission Lagarrigue -...). Les associations de spécialistes jouent un rôle pilote dans chacun de leurs domaines, comme l'AFPF puis l'AFEF ${ }^{2}$ pour le français, l'APM pour les mathématiques, l'Union des physiciens... et des équipes de didactique sont constituées dans quelques universités, en relation étroite avec une discipline.

\section{La recherche en éducation : « théorie » et «pratique»}

4 La très vive polémique qui se développe alors (dans l'atmosphère de 1968 et de la réaction qui s'ensuivit) a une dimension clairement politique, les contempteurs de la « rénovation » se situant très majoritairement à droite. La chose n'était pas nouvelle, et sans même remonter à la Monarchie de Juillet, à l'époque de Jules Ferry, à celle de l'avant-première-guerre mondiale, ou des années 1930, on retrouvait, avec les mêmes arguments, la véhémence qui sera celle des adversaires du Plan Langevin-Wallon de l'époque de la Libération : c'étaient les changements annoncés, et donc non encore mis en œuvre, qui menaçaient, puisqu'ils mettaient en cause une tradition insoupçonnable par 
définition. La polémique rebondissait avec l'arrivée de la Gauche unie au pouvoir après 1981, le rapport Carraz de 1983 sur «La recherche en éducation et la socialisation de l'enfant ", en étant la défense et illustration.

On trouve en effet dans les débats que ce rapport suscite alors tous les ingrédients encore aujourd'hui présents dans le paysage: doutes sur la possibilité de recherches en éducation, primat de la "pratique » sur toute "théorie », éventuellement considérée comme impossible ou inconsistante, d'un côté; de l'autre recours aux arguments venus de la tradition souvent relayée du modèle ecclésiastique, que résument l'alpha et l'oméga des qualités du "bon maître »: "avoir la vocation", "avoir du charisme », avec ses déclinaisons pouvant confiner au bout du compte à l'obscurantisme le plus contradictoire avec le souci affirmé par les mêmes de l'importance du « savoir ».

6 On pourrait résumer la configuration des relations entre niveaux d'enseignement, formation et recherche par le tableau suivant, qui montre que la position globale des IUFM incite au bousculement des catégories issues de traditions séculaires :

\begin{tabular}{|c|c|c|c|}
\hline $\begin{array}{l}\text { Recherche } \\
\text { en éducation }\end{array}$ & Primaire & Secondaire & $\begin{array}{l}\text { Technique et } \\
\text { professionnel }\end{array}$ \\
\hline $\begin{array}{l}\text { Publics } \\
\text { scolaires }\end{array}$ & Publics scolaires & $\begin{array}{l}\text { Enfants de l'élite } \\
\text { socio-économique }\end{array}$ & $\begin{array}{l}\text { Enfants de milieu } \\
\text { populaire }\end{array}$ \\
\hline $\begin{array}{l}\text { Point de } \\
\text { vue des } \\
\text { contemp- } \\
\text { teurs de la } \\
\text { recherche } \\
\text { en éduca- } \\
\text { tion. }\end{array}$ & $\begin{array}{l}\text { Inutile et inexistante, il suffit de } \\
\text { bons maîtres et de bonnes pra- } \\
\text { tiques à reproduire. Forma- } \\
\text { teurs de terrain seuls indispen- } \\
\text { sables. }\end{array}$ & $\begin{array}{l}\text { Inutile; la recherche } \\
\text { ne peut concerner } \\
\text { que les contenus } \\
\text { d'enseignement. } \\
\text { Formateurs de ter- } \\
\text { rain nécessaires, } \\
\text { après les formateurs } \\
\text { universitaires. }\end{array}$ & Inutile \\
\hline $\begin{array}{l}\text { Point de vue } \\
\text { des parti- } \\
\text { sans de la } \\
\text { recherche } \\
\text { en éduca- } \\
\text { tion. }\end{array}$ & $\begin{array}{l}\text { Nécessaire, d'autant qu'est } \\
\text { visée la réussite pour tous les } \\
\text { élèves, le destin scolaire étant } \\
\text { déjà socialement tracé à la } \\
\text { fin de l'école primaire; refus } \\
\text { d'opposer «théorie » et "pra- } \\
\text { tique " (" académique " et } \\
\text { " professionnel "); étudier les } \\
\text { rapports entre apprentissages } \\
\text { scolaires disciplinaires, savoirs } \\
\text { savants, savoirs enseignés, } \\
\text { savoirs scolaires ; mise en } \\
\text { cause de la polyvalence indivi- } \\
\text { duelle des maîtres, considérée } \\
\text { comme une fiction. } \\
\text { Formateurs diversifiés : ensei- } \\
\text { gnants chercheurs, enseignants } \\
\text { de terrain... } \\
\text { Travail collectif des maîtres } \\
\text { entre eux, des maîtres et des } \\
\text { parents, des maîtres et des } \\
\text { élèves... }\end{array}$ & $\begin{array}{l}\text { Nécessaire, en parti- } \\
\text { culier pour sortir } \\
\text { des échecs sociale- } \\
\text { ment inégaux ; lien } \\
\text { entre primaire et } \\
\text { secondaire à étudier, } \\
\text { comme entre } \\
\text { secondaire et supé- } \\
\text { rieur. } \\
\text { Formateurs : diversi- } \\
\text { fiés comme en pri- } \\
\text { maire. }\end{array}$ & $\begin{array}{l}\text { Nécessaire, pour } \\
\text { combattre l'usage } \\
\text { du technique-pro- } \\
\text { fessionnel comme } \\
\text { voie de relégation. } \\
\text { Formateurs : diver- } \\
\text { sifiés comme en } \\
\text { primaire et secon- } \\
\text { daire. }\end{array}$ \\
\hline
\end{tabular}

7 Le débat dispose en effet d'un arrière-plan qu'il serait naïf de négliger. Le rapport à la recherche est à considérer en relation avec le rapport à la pratique : la vision dominante encore était l'opposition théorie/pratique, faisant référence à des traditions à la fois différentes et parallèles entre primaire et secondaire, dans la mesure où la connivence sociale propre au secondaire, réservé en effet jusqu'en 1975 (création du «collège unique») aux enfants des milieux sociaux les plus favorisés, mettait l'accent bien davantage sur les contenus d'enseignement, destinés non à tous, mais à la seule " élite » future. En somme, au secondaire de l'élite la «théorie», au primaire pour tous la " pratique ». Mais quid quand la scolarité obligatoire était prolongée dans le secondaire? Jusqu'en 1972, et la généralisation d'un recrutement après le baccalauréat, les écoles normales d'instituteurs, comme les écoles normales supérieures pour les professeurs, s'étaient peu intéressées à la pédagogie, occupées qu'elles étaient à donner accès au 
niveau d'études destiné à obtenir le diplôme ouvrant la voie aux certifications des connaissances disciplinaires correspondantes.

Certes, le rapport à la recherche est une des orientations de l'Éducation nouvelle à partir de Binet avant la Première Guerre mondiale, reprise ensuite par le Groupe Français d'Éducation Nouvelle, qui sera l'inspirateur principal du Projet de réforme LangevinWallon (1947). La mention du besoin de recherches pédagogiques (au pluriel le plus souvent) y est souvent citée : ainsi indique-t-il que les écoles normales (destinées aux maîtres non spécialisés du primaire) sont des lieux de recherche, que leurs enseignants sont formés à la recherche, que les inspecteurs (recrutés à partir de dix ans d'enseignement) sont en rapport avec la recherche...

La boussole que constitue le Plan pour les différents courants progressistes en éducation explique la constance pour leur part de l'importance accordée à la recherche. La création des sciences de l'éducation ${ }^{3}$ en 1965-67 (Debesse et Mialaret), les travaux autour du Plan de rénovation en français (1967-72), des IREM en mathématiques, etc. où l'IPN, puis l'INRP, jouent un rôle décisif, avec reprise après 1981, sont soutenus par les organisations syndicales les plus représentatives, comme par les associations de spécialistes par discipline, en dehors de celle des professeurs de philosophie.

La création des IUFM dans le cadre de la loi d'orientation Jospin (1989) est vécue comme un acquis de ces courants progressistes. Les attaques viennent toujours de droite et d'extrême droite ${ }^{4}$, mais aussi d'un certain nombre d'universitaires, au nom d'une critique sans nuance des travaux qui s'intéressent à la relation au savoir des élèves, désormais articulés autour du nouveau champ des didactiques ${ }^{5}$. Elles s'appuient aussi sur une forte méfiance vis-à-vis de la discipline "sciences de l'éducation » telle qu'elle est présente dans les universités et au CNU. La commission Bancel, préalable à la loi d'orientation, confère non sans débats aux IUFM un rôle de participation à la recherche. Les aléas des politiques ultérieures ne manquent pas d'affecter la conception de la recherche en IUFM, en relation avec le nombre des postes d'enseignants chercheurs de ces établissements, plutôt limités, et des moyens dégagés à cet effet, en particulier pour décharger les personnels de second degré en vue de préparer des thèses, et/ou de participer à des recherches. Le pilotage de celles-ci va aussi connaître des fluctuations, des tentatives de liaison au niveau national (en particulier en relation avec l'INRP, lui-même amené à « s'universitariser ») à la recherche de liens avec le milieu universitaire local.

\section{Les solutions mises en place 1990-1995: de la nébuleuse à la dynamique}

11 Dans ce contexte compliqué d'un « chemin montant, sablonneux, malaisé » comme aurait dit La Fontaine, l'émergence de la recherche n'a pas été sans cahots dans les IUFM. On en dégagera ici les caractères communs et les principales solutions, qui ont pu être repérés et analysés in vivo grâce à une recherche sous l'égide de la DRED, coordonnée par Lise Demailly et Danielle Zay, et qui a fait l'objet d'un rapport paru en 1993 et d'articles dérivés, cités en bibliographie. 


\section{1. Caractères communs}

12 A/ L'affirmation générale concernant la recherche résulte de la loi d'orientation de 1989 qui stipule que "les IUFM participent à la recherche en éducation", formulation vague mais prudente. À partir de là, on est dans la variation et le flou. Tout est dans l'interprétation de "participent à » ainsi d'ailleurs que de ce qu'on entend par « recherche en éducation».

L'interprétation généralement admise dans les universités renvoie la recherche du côté universitaire, les IUFM pouvant, par le biais des conventions qui les lient aux universités, y prendre part. Certains IUFM toutefois cherchent à "participer » à la recherche en favorisant l'émergence en leur sein de centres de recherche (le terme d'équipe étant pour l'heure réservé aux universités et grands organismes).

Quant à "recherche en éducation ", c'est une expression floue, qui peut être interprétée de bien des façons: recherche du domaine des sciences de l'éducation, recherche en didactique des disciplines, recherche pédagogique, recherche-action, innovation, expérimentation... La formulation «recherche en éducation » évite justement celle de " sciences de l'éducation ", vis-à-vis desquelles les porteurs des IUFM, porteurs politiques et administratifs, voire universitaires, marquent une certaine défiance. Par ailleurs un organisme comme la MAFPEN (Mission académique de formation des personnels de l'Éducation nationale), chargé alors de la formation continue des enseignants dans chaque rectorat, peut ainsi organiser des actions dites de "recherche», mais sans base documentaire ni méthodologie précise ni évaluation. Un des avantages de l'avancée de la recherche dans les IUFM sera d'ailleurs de mettre au clair ce qu'on entend par là, au plan méthodologique notamment.

Enfin, le rôle attribué à la recherche en IUFM est ambigu : en dehors du fait qu'il s'agit de donner une légitimité universitaire à l'institut, la recherche sert-elle à la formation des formateurs, à la formation des étudiants et stagiaires, à l'amélioration de l'enseignement scolaire ? Les débats seront fréquents et animés sur ce sujet pendant plusieurs années.

13 B/ Le flou se retrouve dans les modalités de la mise en place, qui va se faire par tâtonnements successifs. Il n'y aura pas de modèle imposé d'en haut, des solutions locales vont s'inventer IUFM par IUFM (certains IUFM d'ailleurs n'inventeront pas de solution du tout dans les premières années). Les facteurs qui vont jouer un rôle majeur sont l'environnement universitaire plus ou moins riche, plus ou moins accueillant ou hostile à l'IUFM aussi, le nombre d'enseignants-chercheurs dans l'institut, le volontarisme du directeur de l'Institut et sa position scientifique par rapport aux universités, la position du responsable à la recherche (s'il y en a un) par rapport aux équipes universitaires, la présence d'une tradition de recherche ou pas dans l'école normale d'instituteurs qui a précédé l'IUFM.

Cette dispersion, maximale pendant les deux premières années, va progressivement se réduire, d'une part par des réunions nationales des responsables à la recherche dès 1991, d'autre part par une coordination plus ferme entre les directeurs d'IUFM sur cette question à partir de 1993.

$14 \mathrm{C} / \mathrm{La}$ mise en place de la recherche en IUFM a eu à s'adapter aux évolutions politiques nationales, en accordéon. Si dans un premier temps, dans la foulée du rapport Bancel, les IUFM étaient incités à s'intéresser à la recherche en éducation, à partir de 1993 et du gouvernement Balladur, un coup d'arrêt est donné et François Fillon, alors ministre, 
affirme publiquement que « les IUFM ne sont pas le cadre organisateur de la recherche, même pédagogique » (01/07/93) (Demailly \& Zay, 1997 : 17). Mais deux ans plus tard, les positions seront à nouveau assouplies.

\section{2. Quelques solutions variées}

15 Demailly et Zay (1997) établissent une typologie des « services recherche » mis en place par les IUFM et distinguent six cas de figure, correspondant selon elles à des logiques différentes :

- la logique de laboratoire ;

- la logique de réseau ;

- la logique de soutien à l'innovation ;

- la logique de sujétion à la recherche universitaire ;

- la dispersion par manque de ressources ;

- la dispersion en nébuleuse.

Ce classement, basé sur des monographies complètes fournies par 14 IUFM (sur 26) et des informations partielles apportées par quelques autres, a le mérite de faire apparaître les diversités de solutions qui s'ouvrent alors aux Instituts, lesquels ont beaucoup de problèmes à régler en même temps. Mais le souci de la modélisation, comme souvent, écrase un peu la diversité du réel. Et un même IUFM peut mettre en œuvre des solutions empruntées à deux ou plusieurs des logiques énumérées, comme on le verra dans l'exemple de Montpellier.

17 On n'entrera pas ici dans une étude quantitative des moyens, qui mériterait de faire l'objet d'une présentation détaillée. Par exemple, la faiblesse du nombre des postes d'enseignants-chercheurs en 1992, an 1 des IUFM, est une donnée fondamentale : 2776 emplois de second degré, 486 d'enseignants chercheurs, soit $15 \%$, mais les uns ayant un service horaire double des autres, cela ne représentait que moins de $6 \%$ devant les étudiants ${ }^{6}$.

Parmi les moyens mis en place par tel ou tel Institut on notera en vrac:

- la désignation d'un responsable recherche, chargé de cette question au sein de l'IUFM ; son statut est variable : parfois simple chargé de mission, parfois directeur-adjoint, il pourra ensuite se confondre (ou pas) avec le président du CSP, Conseil Scientifique et Pédagogique ; le groupe national des responsables à la recherche, seul lieu de contact entre les IUFM sur cette question au début, sera dissous en 1995 à la demande des directeurs, désireux d'être seuls pilotes de ce domaine ;

- la création d'une ligne budgétaire affectée à la recherche, là aussi d'ampleur variable ;

- la création d'un Centre de recherche (rare) propre à l'Institut et distinct des équipes de recherche universitaires;

- la tenue de journées d'études, colloques, séminaires ;

- des appels d'offre recherche sur des thèmes prédéfinis par l'institution, permettant de faire travailler ensemble des personnels de statut différent ;

- la participation des enseignants-chercheurs des IUFM aux équipes universitaires ;

- un travail commun entre chercheurs des universités et enseignants-chercheurs des IUFM ;

- un travail commun avec les MAFPEN et les corps d'inspection;

- un encouragement à la réponse aux appels d'offre INRP ;

- une incitation des formateurs IUFM à la préparation de DEA et thèses sur des sujets relatifs à l'éducation et à la didactique ; 
- la création de revues propres à l'Institut ;

- la création de bases documentaires, bibliothèques...

\section{3. Un exemple parmi d'autres : Montpellier}

\section{Le contexte}

Placé dans un environnement universitaire riche, l'IUFM de l'académie de Montpellier prend le relais d'écoles normales qui possèdent une forte tradition de recherche INRP dans deux de ses centres majeurs: Nîmes et Montpellier. La création de l'IUFM a été précédée de réflexions sur la formation des enseignants au sein de l'université Paul Valéry (Lettres et Sciences humaines), dans le département de Formation à l'intérieur des Sciences de l'Éducation. Il existe des recherches en didactique au sein des universités (sciences du langage, anglais, sciences physiques, IREM). Le directeur de l'IUFM et la responsable à la recherche, tous deux venant de l'université littéraire, ont une forte implantation de chercheurs, et des liens anciens avec les écoles normales, la MAFPEN ou l'INRP).

Toutefois l'accueil universitaire vis-à-vis de l'IUFM est variable selon les universités : correct et participatif de la part de l'université des sciences, il est rageur et très polémique de la part de l'université littéraire, qui au début critique vivement à peu près systématiquement tous les projets.

\section{Les actions}

21 Dès la création en septembre 1991 le directeur de l'IUFM souhaite développer la recherche et désigne pour cela un (e) responsable, professeur d'université.

La mission est de développer dans l'IUFM une recherche qui place l'Institut à égalité avec les universités, tout en affichant l'originalité de l'institution et de ses tâches. La mission va se décliner de différentes façons.

\section{Des instruments autonomes}

L'IUFM s'efforce de créer le plus tôt possible un centre de recherches interne à la maison, de type scientifique, qui affirme la légitimité de la recherche en IUFM face aux universités. C'est ce que Demailly \& Zay (1997) appellent la logique de laboratoire. Cela va se faire en deux ans. De juin 1990 à septembre 1992, une commission de réflexion sera chargée de l'élaboration d'un document préparatoire sur les grandes lignes du Centre ce qui aboutira à une charte provisoire. En 1992, le centre ouvrira sous l'appellation CEDRE (Centre d'Études Didactiques et de Recherches sur l'Enseignement). À la différence des laboratoires universitaires, toutefois, le Centre joue la carte de l'interdisciplinarité (notamment en raison du petit nombre d'enseignants-chercheurs qui sont affectés à temps plein à l'IUFM), et de l'intercatégorialité, de façon à faire travailler ensemble chercheurs et praticiens. "Il (le CEDRE) s'efforce de susciter des recherches fondamentales ou appliquées, regroupant les différents degrés, corps et spécialistes de la formation. Associant universitaires et praticiens, collaborant avec les différentes instances de la Recherche, il souhaite jouer un rôle de lieu de rencontre, de discussion et d'incitation sur les problèmes relatifs à l'enseignement, l'apprentissage et la formation » ( Tréma, 1, 1992, p 88). 
23 La création du CEDRE s'accompagne d'une journée d'études sur la recherche et la formation des maîtres, le 5 février 1992. Appelée Forum-recherche, elle a pour objectif de sensibiliser les diverses catégories de formateurs à l'intérêt de développer la recherche en IUFM. Y interviennent enseignants-chercheurs de l'IUFM et de l'université, formateurs de second degré, corps d'inspection. Elle sera suivie en 1993-1994 de la création d'un séminaire méthodologique, qui a lieu à l'IUFM à Montpellier, destiné à former à la méthodologie de la recherche.

Très vite également se met en place une publication. L'IUFM à Montpellier a été le premier à créer une revue de recherche, la revue Tréma, dont le premier numéro paraît en mai 1992. Son objectif affiché est d'« articuler réflexions scientifiques, recherche sur l'enseignement et formation des enseignants ", (Tréma 1, 1992, p. 1) selon la logique IUFM du CEDRE. Le caractère scientifique assumé (qui conduira plus tard cette revue à la reconnaissance AERES), et toujours l'interdisciplinarité associée à la diversité des auteurs, la distinguera d'autres revues IUFM à vocation plus nette de formation pédagogique. Cette revue a produit 38 numéros plus deux hors série en vingt ans.

Dernier volet du dispositif CEDRE, la création d'un Fonds documentaire en Histoire de l'Éducation en septembre 1992, (devenu Centre d'Études, de Documentation et de Recherche en Histoire de l'Éducation, CEDRHE) grâce à son fondateur Pierre Guibbert, qui fait un don initial de 3000 ouvrages, manuels scolaires notamment. Il assurera ensuite la responsabilité du Fonds, son développement, son catalogage, les relations avec d'autres fonds comme celui de l'INRP ou du Georg-Eckert-Institut de Braunschweig. Le Fonds permettra l'organisation d'expositions en partenariat avec la DRAC et la ville de Montpellier, ou de débats à l'IUFM.

\section{Des actions de développement de la recherche en réseau}

Prenant en compte sa situation particulière, entre rectorat et universités, l'IUFM lance un appel d'offres de recherche académique, sur thèmes imposés, en direction d'équipes intercatégorielles de formateurs IUFM.

Les contacts sont pris avec la MAFPEN, où les IPR et certains IEN organisent des actions de recherche tournées vers une pratique professionnelle ; avec l'INRP, de façon à ce que des formateurs IUFM participent aux recherches nationales en échange d'heures de décharge; avec l'IREM auquel collaborent plusieurs formateurs de mathématiques, y compris enseignants-chercheurs (il est basé à l'Université des sciences). On est là dans ce que Demailly \& Zay appellent la logique de réseau, qui va se renforcer progressivement, lorsque la responsable à la recherche des débuts deviendra directrice-adjointe en 1995 puis chargée de la formation continue académique pour le maitre d'œuvre IUFM. Les groupes d'études et de recherche (GER) mis alors en place en 1997-1998 seront l'exemple de cette mise en réseau des divers organismes participant à la formation, comme le souligne le rapport Prost en 2001.

\section{L'effort en direction des universités}

Les instruments spécifiques créés par l'IUFM afin de développer la recherche en éducation n'ont jamais signifié la volonté de se couper des universités. C'était de toute manière impensable, compte tenu de l'affirmation sans cesse répétée de la primauté universitaire de la recherche, et encore plus de 1993 à 1995, quand les IUFM se sont trouvés « interdits » de recherche. Les enseignants-chercheurs à Montpellier ont donc été 
encouragés à faire partie des équipes universitaires ou du CNRS. Pour Demailly \& Zay, cela relèverait d'une troisième logique, appelée « sujétion universitaire ».

Dans ce dernier cas, le CNRS ignorant dans l'ensemble les didactiques et les recherches en éducation, les recherches ne pouvaient être que de type fondamental. En université, certains chercheurs se sont battus pour que la didactique devienne un volet des programmes de recherche des équipes. Ce fut le cas en lettres pour l'équipe DIPRALANG, équipe d'accueil, dont le volet didactique établi clairement en 1995 sous l'appellation Didaxis permettra ensuite qu'elle devienne en 1998 équipe mixte université-IUFM, et qui a accueilli ainsi les enseignants-chercheurs de français de l'IUFM. Là encore l'évolution a été facilitée par le fait que la responsable à la recherche de l'IUFM est devenue en 1995 directrice de l'équipe DIPRALANG, et membre des grands conseils de l'université. Ce fut le cas aussi en sciences, où une composante didactique a pu se constituer petit à petit en sciences physiques notamment. Ce mouvement a permis la mise en place de séminaires de DEA dès 1993 en université littéraire, la préparation de DEA et de thèses.

Dans les débuts de son existence, l'IUFM de l'académie de Montpellier s'est donc efforcé d'utiliser différents moyens pour développer la recherche en éducation, en affirmant sa légitimité comme lieu de recherche, en cherchant à créer des relations avec les partenaires non universitaires de la formation, et en maintenant voire en resserrant les liens avec les équipes universitaires. Il faut bien dire que c'était un peu jouer la quadrature du cercle, et les obstacles se bousculaient à chaque pas.

\section{D'hier à aujourd'hui}

Les enjeux présents à l'origine ont-ils trouvé un aboutissement ?

Les caractères de la recherche en IUFM tels qu'ils sont exposés dans le rapport Prost de 2001 montrent qu'ils étaient en germe dans les solutions mises en œuvre par les IUFM en matière de lancement de la recherche, dans les années 1990-1995 :

- la dispersion des recherches en éducation;

- la variété des actions proposées, liée à celle des relations avec le monde universitaire selon l'environnement ;

- le souci de valoriser la recherche en éducation sous toutes ses formes ;

- l'équilibre dans les recherches en didactique entre le scientifique et le souci de formation pratique.

31 Surmontant les réticences, les IUFM se sont depuis dotés d'équipes spécifiques ; dès 2001 l'annuaire thématique « Recherche en éducation et formation » réalisé par la Conférence des Directeurs d'IUFM (4e édition, Juin 2001) présente 61 équipes, sous des dénominations diverses (pôles, thèmes, axes, opérations). (Prost, 2001, p. 10).

On pourrait penser que l'inscription des IUFM dans le champ universitaire de la recherche a réussi en ce sens que des équipes spécifiques ont été créées, puis que les Instituts ont été absorbés par les universités. Par contre, la distance par rapport aux sciences de l'éducation, forte dans les débuts des IUFM, a fondu, bon nombre de ces équipes relevant désormais de ce domaine. Peut-être les trois faits sont-ils liés.

Mais si les formes ont changé, les thèmes de travail également, les options restent peutêtre globalement identiques.

Le feu roulant de critiques concernant les IUFM dès leur création concerne au premier plan justement leur prétention à la recherche. On y trouve, ou y retrouve, pêle-mêle, le 
vieux mépris de "ceux qui savent " parce qu'ils s'adressent à des élèves plus âgés, par rapport à " ceux qui n'ont pas besoin de savoir ", puisqu'ils s'adressent à de très jeunes élèves (les «primaires ») ; la défiance à l'égard de la nouveauté, censée mettre en cause une "tradition qui a fait ses preuves»; le refus de prendre en compte l'historicité des disciplines scolaires : or celle-ci montre facilement qu'il s'agit de constructions liées à des aléas multiples, mais sûrement pas à une rationalité intrinsèque.

Pourtant c'est le moment où un certain nombre de travaux renouvellent la relation entre "savoir savant", «savoir scolaire", "savoir enseigné", pour mieux comprendre les « rapports au savoir » d'élèves désormais considérés dans leurs difficultés, non réduites à des insuffisances morales ou intellectuelles. C'est le moment où A. Chervel montre que les savoirs savants ne sont pas indépendants des développements des savoirs scolaires, où J.L. Martinand fait valoir que les disciplines sont soumises aussi à des «pratiques sociales de référence ", où Y. Chevallard invite à s'intéresser aux transpositions didactiques nécessaires pour choisir ce qui peut et doit être enseigné, en fonction des cursus, où se développent les recherches liées à la mise en œuvre de la découverte des travaux de Vigotski, et à ceux de la littératie (soit la prise en compte des activités de lecture-écriture dans le développement des cultures et des cursus).

Niant en bloc ces réflexions, les adversaires des IUFM s'installent dans une position où la théorie et la pratique ne peuvent avoir de relations, et en particulier se penser : il y aurait d'un côté des savoirs, de l'autre des pratiques ${ }^{7}$. L'amélioration dans ces conditions ne pouvait venir que de l'accroissement des savoirs enseignés (qui ne sont pas interrogés quant à leur choix ou à leur utilité), ou/et de la recherche de la «bonne pratique » (pas plus soumise à évaluation sérieuse) ${ }^{8}$, reproduite par simple mimétisme, combinée de préférence à un "charisme» associé à une "vocation», dont le défaut éventuel expliquerait l'échec du maître, à moins que ce ne soit celui d'élèves «non doués » ou handicapés socio-culturels.

Il est vrai que de leur côté, les premiers partisans de la « rénovation » des années 1960, reprenant d'ailleurs les thèses de l'Éducation nouvelle, qui avaient pignon sur rue depuis les années 1930 (Gutierrez, L., Besse, L, Prost, A. dir., 2012), avaient considéré comme allant de soi qu'il existait un enseignement «traditionnel » uniforme, et qu'il suffisait pour l'essentiel d'appliquer les nouvelles données théoriques issues notamment de la linguistique, à une époque où le structuralisme semblait favoriser les transferts interdisciplinaires.

36 L'échec de la mise en place, sans doute trop hâtive, de modifications d'importance dans les contenus en mathématiques (les "maths modernes»), à un degré moindre en grammaire, la sous-estimation des résistances des pratiques des maîtres, loin d'être toutes injustifiées, vont fournir aux adversaires du changement un terrain d'approche favorable. Au grand colloque de Marseille de 1997, les chercheurs en éducation de tous horizons plaidèrent avec force pour que soient mis au plus vite à la disposition de tous les moyens offerts par la recherche en vue de sortir d'une crise commençant à affecter les progrès d'un accès plus démocratique aux études. Le sursis sera de courte durée.

Le changement politique à droite à partir de 2002 aboutit à l'impensable dix ans plus tôt. Dans une période de réaction historique, la recherche en éducation devient, avec les IUFM, une des cibles privilégiées des critiques : la solution désormais était de revenir en arrière, puisque le mal venait d'avoir voulu changer la tradition'. C'était l'inversion du rapport nouveau=bon / tradition=mauvais. 
situation de juin 2012 est notamment marquée par un effondrement sans précédent du nombre des candidats aux concours de recrutement, mais aussi l'abandon de la réflexion sur les nouvelles formes des différents métiers de l'enseignement. Elle oblige à s'interroger sur les conditions à remplir pour assurer le renouveau et le développement urgent et indispensable des recherches en éducation: implication de la profession, mobilisation des moyens en personnels et en fonctionnement, sans préjudice pour les autres domaines de recherche, programmes nationaux de recherche ambitieux et cohérents, information en direction des parents, du public en général, soumis depuis plus de vingt ans à un grand nombre de pamphlets souvent peu scrupuleux, pour le moins, complaisamment relayés par des médias peu critiques.

montre en mars 2013 le récent accroissement du nombre des postes, qui entraine un accroissement du nombre des candidats, la question des moyens est évidemment décisive.

En tous les cas, des centres de formation des maîtres ne sauraient, pas plus que le monde universitaire dans son ensemble, se priver d'une relation forte à la recherche en éducation, sous toutes ses formes; encore faut-il donner du temps à l'initiation à la recherche dans les cursus de formation.

\section{BIBLIOGRAPHIE}

Boutan, P., Sorel, E. (dir.), Le plan Langevin-Wallon une utopie vivante, Paris : PUF, 1998.

Boutan, P. « Histoire de la formation des maitres et enseignement de la langue nationale ", communication, Colloque Défendre et transformer l'école pour tous, Marseille, 3-5 octobre 1997 (cédérom IUFM d'Aix-Marseille), 1997.

Boutan, P. (avec G. Bouchet et F. Bouillon), « Continuité et changement en matière de réforme et de professionnalisation de la formation des maîtres en France ", communication, Biennale de l'éducation et de la formation, Paris, du 18 au 21 avril 1996 (édité sous cédérom), 1996.

Demailly, L. et Zay, D. coord., Emergence des politiques de recherche dans les IUFM. Étude de la mise en place d'unités organisationnelles chargées de la recherche au sein des IUFM (septembre 1990-décembre 1992). Rapport DRED, Paris, 1993.

Demailly, L. \& Zay, D., « Gestion de la recherche et formation de formateurs d'enseignants ». Recherche et formation. Paris : INRP, n¹7, 1994, p. 47-64.

Demailly, L. \& Zay, D., « Politiques et organisations dans la recherche-développement : le cas des instituts Universitaires de Formation des maîtres ». Revue française de pédagogie. Paris : INRP, $\mathrm{n}^{\circ}$ 121, oct-dec 1997, p. 79-97.

Former des Maîtres, bulletin du Syndicat national des professeurs d'école normale (SNPEN), puis du Syndicat national des professeurs d'IUFM, puis du SNESUP. En particulier : $\mathrm{N}^{\circ} 152$, juin 1983, «Compte rendu du carrefour de Sèvres : ‘Former des maitres pour l'an 2000' ». N²2, mai 1993. N • 43, janvier 1995. N 44, février 1995. 
Gutierrez, L., Besse, L, Prost, A. (dir.)., Réformer l'école. L'apport de l'Éducation nouvelle (1930-1970). Grenoble : PUG, 2012.

Jacquet-Francillon F., d'Enfert R., Loeffel L. (dir.), Une histoire de l'école. Anthologie de l'éducation et de l'enseignement en France XVIIIe-XXe siècle. Paris : Retz, 2010.

Lapierre, G. (dir.)., Qui forme les enseignants en France aujourd'hui ? Grenoble : université Pierre Mendès France, 1996.

Verdelhan-Bourgade, M., L'apport original des IUFM à la recherche en éducation. Colloque Recherche et formation des enseignants, IUFM, Toulouse, 1995.

\section{Textes et rapports officiels}

Loi d'orientation n89-486, 10 juillet 1989, article 17.

Bancel, Daniel., Créer une nouvelle dynamique pour la formation des maîtres. Rapport à Lionel Jospin, Ministre de l'Éducation nationale, de la Jeunesse et des Sports. Paris, La documentation française, 1989, 19 p. http://formerlesformateurs.free.fr/rapport_bancel_10oct2006.html

Carraz, Roland., Recherche en éducation et socialisation de l'enfant. Rapport de mission au ministre de l'Industrie et de la Recherche, Paris, La documentation française, 1993.

Caspar, Pierre., La formation des formateurs en IUFM. Réflexions sur la formation des formateurs en IUFM . Rapport remis au Ministre de l'Éducation nationale, 2002, 48 p.

Décret n 90-967 du 28/09/1990, fixant les règles d'organisation et de fonctionnement des instituts universitaires de formation des maîtres. Rôle du Conseil scientifique sur la recherche en éducation.

Kaspi, A., Rapport sur les instituts universitaires de formation des maîtres. Rapport à M. le ministre de l'Éducation Nationale, M. Le ministre de l'Enseignement Supérieur de et de la recherche, 1998.

CNE., Les IUFM au tournant de leur première décennie. Panorama et perspectives. Rapport d'évaluation, 2001.

Prost, A., Pour un programme stratégique de recherche en éducation. Rapport remis à MM. Les Ministres de l'Education nationale et de la Recherche, Paris, La documentation française, 2001, 78 p.

\section{NOTES}

1. / Il faut rappeler que c'est seulement au temps de Jules Ferry que tous les futurs professeurs du secondaire durent passer par les facultés de sciences et de lettres, ce qui permit d'ailleurs à celles-ci de connaître une expansion sans précédent.

2. / AFPF : association française des professeurs de français, AFEF : association française des enseignants de français, APM : association des professeurs de mathématiques.

3. / Les premières chaires de Science de l'éducation (au singulier) sont créées dans les universités par Jules Ferry.

4. / Dans un rapport parlementaire de 1986, le député du Front National Jean-Claude Martinez demandait la réduction de l'INRP à trois emplois de bibliothécaires.

5. / Les différents détracteurs des IUFM, en même temps que des sciences de l'éducation en général et des différentes didactiques disciplinaires, se retrouvent dès 1989 sur une première pétition au président de la République lui demandant de renoncer à la nouvelle institution. 
6. / Former des maîtres, $n^{\circ} 22$, mai 1993, p. 15. En 1994, décision est prise de ne plus implanter de postes d'enseignants-chercheurs dans les IUFM. En 1995, 10 postes d'enseignants chercheurs sont créés dans les IUFM (Former des maîtres, $n^{\circ} 43$, janvier 1995, p. 3), pendant que, dans le même temps, l'augmentation des effectifs a été de $37 \%$ en deux ans (Former des maîtres, $n^{\circ} 44$, février 1995, p. 12).

7. / Le rapport signé en 1986 par Hélène Carrère d'Encausse et Jacqueline de Romilly sur «L'Académie française et l'éducation », reprochant leur jargon aux "pédagogues », illustre bien la capacité d'obscurantisme d'une institution héritée de l'Ancien Régime, qui, il est vrai, n'a pas recruté de linguiste depuis plus d'un siècle.

8. / Le livre de François Bayrou, La décennie des mal-appris (1990), est emblématique à cet égard. Trois ans plus tard, quand il est devenu ministre, les IUFM se verront privés de toute relation avec la recherche.

9. / Le comble de cette régression de la plus élémentaire rationalité a été sans doute le financement par le ministre Darcos en 2004 d'une opération de " recherche » visant à mettre en œuvre dans une école primaire les programmes de l'époque de Ferdinand Buisson, sans même que ses protagonistes (dont aucun semble-t-il n'avait une formation à la recherche) se soient préoccupés le moins du monde des études historiques existantes sur le sujet.

\section{RÉSUMÉS}

Après avoir rappelé le contexte historique de l'émergence des questions de recherche en matière d'éducation, l'article montre l'insertion des IUFM, dès leur création en 1991, dans un processus positif, mais lent et assez chaotique ou contradictoire, visant à dépasser l'opposition théoriepratique très en vigueur à l'époque. Le développement des travaux de recherche en matière de didactique(s) dans les vingt dernières années trouve alors un appui et relais dans la formation des maîtres, en lien avec les associations de spécialistes et les forces et courants progressistes, soucieux de combattre l'échec scolaire d'origine sociale. Dans la diversité des situations des différents instituts, de grandes tendances se dégagent, que l'on peut classer. Le cas de l'IUFM de Montpellier fait l'objet d'un développement particulier, car il associe plusieurs de ces tendances. Après un fort développement de la recherche en IUFM, la critique des instituts entre 2002 et 2012, qui a abouti à leur transformation en ESPE en 2013, peut laisser craindre un retour en arrière quant à la relation entre la formation des enseignants et la recherche en éducation.

This article argues that, after their establishment in 1991, the IUFM have gradually bridged the imagined opposition between theory and practice that fueled their creation in the first place. Over the last 20 years, educational research has both developed and found a home in professional programs for training educators, brining together academics and progressive teachers interested in fighting class-linked academic failure. Different institutions have had different positions relative to this academic trend; this article focuses on the IUFM in Montpellier, which brought together a number of these positions. The article also suggests that recent criticism and ultimately reform (2013) of the IUFM may endanger this important legacy by severing the relationship between professional teacher development and educational research. 
INDEX

Mots-clés : IUFM, recherche en éducation

Keywords : Education research, IUFM

\section{AUTEURS}

\section{MICHÈLE VERDELHAN-BOURGADE}

Professeur émérite, sciences du langage, Université Paul-Valéry Montpellier 3

\section{PIERRE BOUTAN}

Maître de conférences honoraire, sciences du langage, IUFM de l'académie de Montpellier 